\title{
SOME ASPECTS OF THE SURFACE TENSION TEMPERATURE DEPENDENCE OF DROP-LIKE AGGREGATES IN MAGNETIC FLUIDS
}

\author{
A.S. Ivanov \\ Institute of Continuous Media Mechanics of the Ural Branch, RAS, \\ Perm Federal Scientific Research Center, 1 Ak. Korolev str., 614013 Perm, Russia
}

An experimental investigation of the surface tension temperature dependence $\sigma(T)$ of the condensed phase (drop-like aggregates) in magnetic fluids undergoing magnetocontrollable phase transition of gas-liquid type was carried out. Accurate numerical processing of experimental data has revealed an anomalous (if compared to ordinary fluids) behavior of the $\sigma(T)$ function for all tested samples: it enhances with the growth of temperature due to the granulometric aspect of the first-order phase transition in magnetic fluids.

Introduction. It is known since the 1970's that the applied magnetic field $H_{0}$ may cause a reversible first-order phase transition in magnetic fluids, which results in magnetic condensation of colloidal particles in a highly concentrated "liquid" phase which is discretely distributed over the entire volume of the initial fluid sample in the form of drop-like aggregates surrounded by the dilute "gaseous" phase. The formation of drop-like aggregates ( $1 \mu \mathrm{m}$ to $1 \mathrm{~mm}$ in size) resembles the appearance of mist droplets in the humid air as temperature decreases. However, the new condensed "liquid" phase in magnetic colloids significantly differs from the initial sample not just in volume concentration of magnetite $\varphi$ (usually magnetiteoleic acid-kerosene based ferrofluids are discussed). The main peculiarity of the condensed "liquid" phase is that it demonstrates the totally different granulometric parameters than the original sample (see Fig. 1).
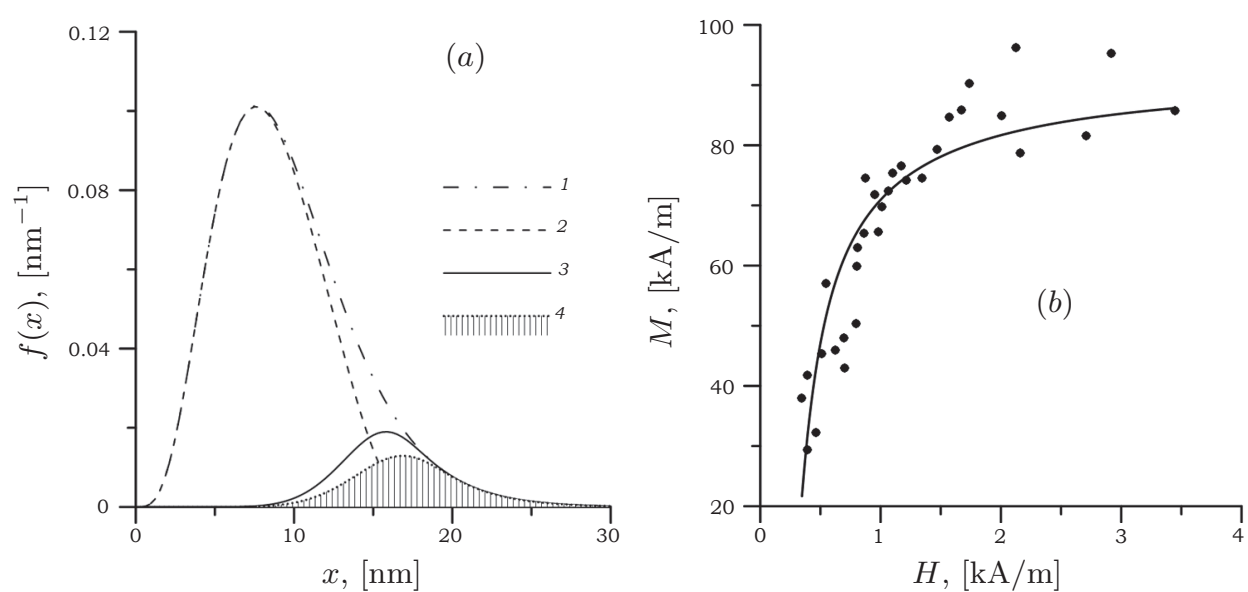

Fig. 1. (a) The typical particle size distribution function of the base colloid $f(x)$ (curve 1), "gaseous" $f_{\mathrm{I}}(x, T)$ (curve 2) and "liquid" $f_{\mathrm{II}}(x, T)$ phases at $T_{1} \approx 20^{\circ} \mathrm{C}$ (curve 3) and at $T_{2} \approx 90^{\circ} \mathrm{C}$ (curve 4). The shaded area under curve 4 is equal to a fraction of large particles capable of aggregating into drop-like aggregates at $T_{2}$. (b) An experimental magnetization curve $M(H)$ for drop-like aggregates at room temperature (reconstructed from [2]). 


\section{A.S. Ivanov}

The explanation is the following: all magnetic fluids are polydisperse (the typical particle diameters $x$ vary in the $3-30 \mathrm{~nm}$ range and the average diameter $\langle x\rangle \approx 10 \mathrm{~nm}$ ), but the "liquid" phase is formed mostly by the largest particles ( $x_{\text {II }}$ varies from 15 to $30 \mathrm{~nm}$ and $\left\langle x_{\mathrm{II}}\right\rangle \approx 20 \mathrm{~nm}$, respectively). Nowadays a numerical technique (verified experimentally) is known [1] which makes it possible to calculate the particle size distribution functions for the "liquid" and "gaseous" phases at a given temperature $T$ if granulometric parameters of the original sample are known (Fig. 1). Large particles which are able to aggregate into drop-like aggregates are not numerous, their volume fraction is about $2-5 \%$ of all colloidal particles in the initial fluid sample, however, the role of this coarse fraction is very important, because the average magnetic moments of the colloidal particles in the initial sample and in the "gaseous" phase, on the one hand, and in the "liquid" phase, on the other hand, differ by one order of magnitude $\left\langle m_{\mathrm{II}}\right\rangle \sim 10\langle m\rangle$, due to the fact that the magnetic moment of a single-domain particle is proportional to its volume, i.e. $m \sim x^{3}$. This circumstance determines the dominant role of the coarse fraction, constituting the "substance" of the drop-like aggregates which are characterized by a surface tension $\sigma$ between the "liquid" and the "gaseous" phase.

This experimental data is also confirmed by the numerical studies $[3,4]$ performed in the framework of the bidisperse ferrofluid model. The existence of $\sigma$ at the 'gas-liquid' interface reminds of ordinary one-component fluids demonstrating a surface tension due to intermolecular attractive forces. However, along with the fundamental difference in nature of the surface tension in magnetic and in ordinary fluids, there is another significant difference - the temperature behavior of $\sigma$. Ordinary one-component fluids demonstrate a strong decreasing $\sigma(T)$ dependence, because the thermal motion disturbs the intermolecular attraction. The $\sigma(T)$ behavior in magnetic fluids is much more complicated because of the granulometric aspect of the first-order phase transition. That is why the present paper is devoted to the investigation of the $\sigma(T)$ dependence of drop-like aggregates.

The last problem to be mentioned in this section concerns the calculation of the magnetization curve $M(H)$ of the condensed "liquid" phase. Due to the high concentration $\varphi$ and large particle diameters $x$, the $M(H)$ curve demonstrates a fast growth and saturation already at small $(\sim 0.5 \mathrm{kA} / \mathrm{m})$ magnetic field intensities (see Fig. 1, right panel). In the framework of the second-order modified mean field theory [5], the $M(H)$ curve can be calculated for any ferrofluid if the explicit form of the particle size distribution function $f_{\mathrm{II}}(x, T)$ is known [1].

1. Experiment and results. The experimental setup was arranged in a standard way: a light-transparent thermostatted Hele-Shaw cell was placed horizontally in the working space of Helmholtz coils, as shown in Fig. 2. An applied uniform magnetic field caused a first-order field-induced phase transition in a thin $(\approx 0.1 \mathrm{~mm})$ layer of the ferrofluid sample. The drops of the condensed phase stretched along the magnetic field lines (Fig. 2). The process of formation and elongation of drop-like aggregates was observed with the help of a video camera integrated into the instrumental microscope head. The temperature of the magnetic fluid sample was controlled by a water thermostat MLW UH in the $15-90^{\circ} \mathrm{C}$ range. The current temperature of the sample was measured by two copper-constantan thermocouples.

Four dilute $(\varphi \approx 0.02)$ magnetic fluid samples with wide and narrow $f(x)$ distribution functions were used in the experiments. All samples demonstrated a field-induced phase transition. Each sample was tested according to the following procedure: first, at zero applied field $H_{0}=0$ the temperature of the sample was 
Some aspects of the surface tension temperature dependence of drop-like aggregates ...

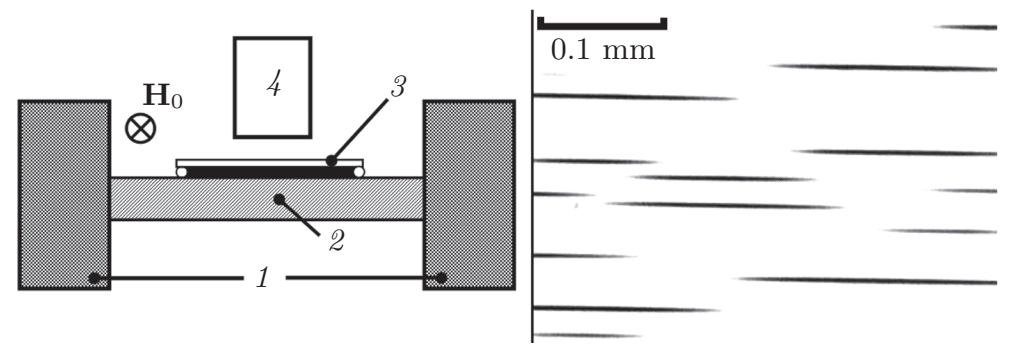

Fig. 2. Left panel: The experimental setup: 1 - heat exchangers, 2 - glass plate, $3-$ cover glass squeezing a wire frame filled with ferrofluid, 4-microscope with a camera, $H_{0}$ - applied magnetic field. Right panel: photo of drop-like aggregates stretched along the magnetic field lines.

set to a new value $T_{i}$, starting from $\approx 18^{\circ} \mathrm{C}$, with a step of $20^{\circ} \mathrm{C}$. At each fixed temperature $T_{i}$, the sample was tested for several applied magnetic field intensities $H_{i}$. Each increase of the field intensity resulted in further elongation of the droplike aggregates in the direction parallel to the field lines. The tested sample at the given $T_{i}, H_{i}$ was captured by the video camera for further processing. When the aggregates grew large and fell out of the camera's observation area, the magnetic field was switched off again and the temperature was increased to its next value $T_{i+1}$. Usually it took about an hour for the system to reach a thermodynamic equilibrium after the temperature increase and about 15 minutes for the relaxation of the drop-like aggregate's shape after the increase of the field intensity $H_{i}$. Upon experiment completion, each snapshot of the Hele-Shaw cell (see Fig. 2, right panel) with the fixed pair of $T_{i}, H_{i}$ values was processed to measure the shape of each aggregate.

The surface tension $\sigma$ of the drop-like aggregates was calculated according to the standard technique [6] that is based on the analysis of the equilibrium aggregate's ellipsoidal shape. The shape depends on the competition between its magnetic energy $E_{\mathrm{M}}$ and its interfacial energy $E_{\mathrm{S}}$. In case both the ellipsoid and the surrounding medium are homogeneous and obey the linear magnetization law (i.e. the magnetization is linearly proportional to the field intensity $M=\chi H$ ), the energies are

$$
\begin{gathered}
E_{\mathrm{S}}=\sigma S=\sigma 2 \pi a^{2} k\left(k+\epsilon^{-1} \sin ^{-1} \epsilon\right), \\
E_{\mathrm{M}}=-\frac{\mu_{0} V_{\mathrm{a}}{H_{0}}^{2}}{2} \frac{\mu_{\mathrm{I}}\left(\mu_{\mathrm{II}}-\mu_{\mathrm{I}}\right)}{\mu_{\mathrm{I}}+\kappa\left(\mu_{\mathrm{II}}-\mu_{\mathrm{I}}\right)},
\end{gathered}
$$

where $S, V_{\mathrm{a}}, \kappa$ and $\epsilon$ are the area, volume, demagnetizing factor and eccentricity of the ellipsoid, respectively; $k=a / b$ is the aspect ratio of the semi-axis, $\mu_{\mathrm{I}}$ and $\mu_{\mathrm{II}}$ are the magnetic permeabilities of the "gaseous" and "liquid" phases, respectively. The equilibrium state is described by the equation $\partial\left(E_{\mathrm{M}}+E_{\mathrm{S}}\right) / \partial \epsilon=0$.

This technique and the system of equations (1) are applicable only for the case of a linearly magnetized medium, i.e. each ferrofluid sample in the experiment should be tested at each pair of $\left(H_{i}, T_{i}\right)$ values with the help of the corresponding magnetization curve: the $(M, H)$ values for the drop-like aggregates with the given $T_{i}$ should not fall out of the linear segment of the curve.

Thus, the $\sigma(H, T)$ dependences for each sample were calculated according to the experimental observations in the framework of the "linear" model (Eqs. 1) (e.g., see Fig. 3). The corresponding $\left.M(H)\right|_{T_{i}}$ magnetization curve calculated at the given temperature $T_{i}$ made it possible to determine the applicability of the "linear" approach in each case. 


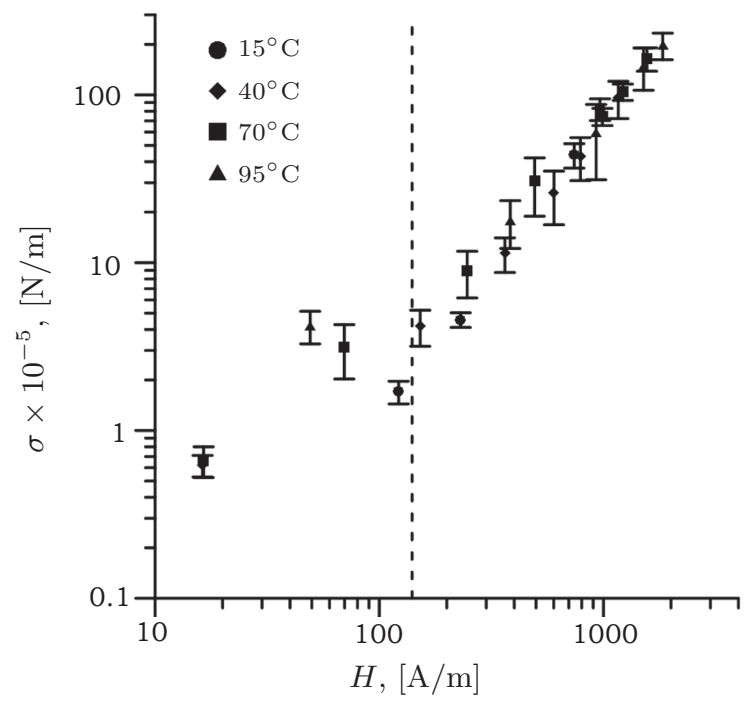

Fig. 3. The "gas-liquid" interface surface tension $\sigma(H, T)$ for the same sample, as in Fig. 1. The dashed line (defined from $M(H)$ curves) divides the plot in two regions, where the linear magnetization approach is applicable (left side) and where it is a priori incorrect (right side).

Fig. 3 shows that only a few first points are reliable, and all the rest are incorrect. Actually, only two samples with wide $f(x)$ functions demonstrated at least several correct $\sigma(H, T)$ values, and the other two samples exhibited the "gasliquid" phase transition at relatively high field intensities $H$, so that the corresponding $\left.(M, H)\right|_{H}$ points fell out of the linear segment of the magnetization curve. However, despite of the limitations of the "linear" theory, it is clear that the surface tension $\sigma$ tends to grow with the increase of temperature: with each fixed $H_{i}$ the field intensity $\sigma\left(H_{i}, T_{1}\right)>\sigma\left(H_{i}, T_{2}\right)$ if $T_{1}>T_{2}$. This extraordinary behavior (if compared to ordinary nonmagnetic fluids) is explained by the granulometric aspect of the "gas-liquid" phase transition in magnetic fluids: the increase in temperature leads to the increase of the dipole-dipole and van der Waals interparticle attraction [4]. Both mechanisms of the interparticle attraction (dipole-dipole and van der Waals) mutually reinforce one another, which results in the experimentally observed increase of $\sigma(T)$.

Acknowledgments. The work was supported by the Russian Science Foundation (project No. 17-71-10010).

\section{References}

[1] A.F. Pshenichnikov, I.Yu. Shurubor. The effect of temperature on the separation of polydisperse magnetic fluids. Magnetohydrodynamics, vol. 24 (1988), no. 4, pp. 417-420.

[2] I.Yu. Shurubor. Stratification of Ferrocolloids: Conditions of Formation and Magnetic Properties of Drop-Like Aggregates (PhD dissertation, Sverdlovsk, 1989) (in Russian).

[3] A.O. Ivanov. Phase separation in bidisperse ferrocolloids. J. Magn. Magn. Matt., vol. 154 (1996), pp. 66-70. 
Some aspects of the surface tension temperature dependence of drop-like aggregates ...

[4] A.O. Ivanov And E.V. Novak. Phase separation of ferrofolloids: the role of van der Waals interaction. Coll. J., vol. 69 (2007), pp. 302-311.

[5] A.O. Ivanov and O.B. Kuznetsova. Magnetogranulometric analysis of ferrocolloids: second-order modified mean field theory. Coll. J., vol. 68 (2006), pp. $430-440$.

[6] J.-C. Bacri, D. Salin and R. Massart. Study of the deformation of ferrofluid droplets in a magnetic field. J. Phys. Lett., vol. 43 (1982), no. 6, pp. 179 184.

Received 27.12.2017 